\title{
ACUPUNTURA COMO TRATAMENTO DA FIBROMIALGIA
}

\author{
ACUPUNCTURE AS TREATMENT OF FIBROMYALGIA
}

\author{
Ana Beatriz Magalhães Brito ${ }^{1}$ \\ Camily Ingrid Araújo Silva ${ }^{2}$ \\ Cristiane Barreto Andrade Ferreira ${ }^{3}$ \\ Laryssa Cristhina Cassimiro Evangelista ${ }^{4}$ \\ Letícia Cristina Araújo Lima ${ }^{5}$ \\ Maria Cristina MartinsMoraes ${ }^{6}$ \\ Silvia Mara Martins ${ }^{7}$ \\ Adamar Nunes Coelho Júnior ${ }^{8}$
}

\begin{abstract}
RESUMO: Caracterizada pela dor crônica, a fibromialgia manifesta seus sintomas de forma constante. Estes causam grande impacto na qualidade de vida do paciente. A mesma vem sendo tratada deforma alopática, porém associada a acupuntura, técnica tradicional chinesa, já sendo possível notar melhoras significativas em seus sintomas. A acupuntura vem se destacando e ganhando adeptos por ser um tratamento não farmacológico. Consiste na estimulação dos pontos energéticos, encontrados em todas as partes do corpo humano. Essa estimulação possibilita a liberação do fluxo de energia acumulada, assim trazendo alívio e diminuição das dores.
\end{abstract}

Palavras-Chaves: Fibromialgia. Acupuntura. Analgesia.

ABSTRACT: Characterized by chronic pain, fibromyalgia constantly manifests its symptoms. These have a great impact on the patient's quality of life. It has been treated allopathically, but associated with acupuncture, a traditional Chinese technique, it is already possible to notice significantimprovements in its symptoms. Acupuncture has been standing

\footnotetext{
I Discente do curso de Biomedicina da Faculdade Única de Ipatinga (FUNIP). Email: annabibs38@gmail.com

${ }^{2}$ Discente do curso de Biomedicina da Faculdade Única de Ipatinga (FUNIP). Email: camilyingrid20or@hotmail.com

3 Discente do curso de Biomedicina da Faculdade Única de Ipatinga (FUNIP). Email: crisbarretoandrade@hotmail.com

4 Discente do curso de Biomedicina da Faculdade Única de Ipatinga (FUNIP). Email:

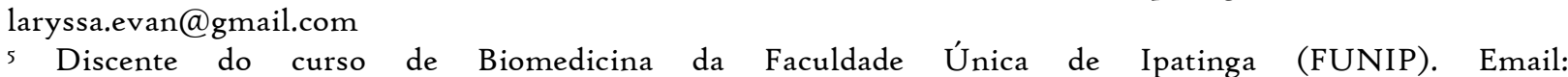

leticialimazoor.lc@gmail.com Biomedicina da Faculdade Única de Ipatinga (FUNIP). Email: mariammoraes6o6@gmail.com

7 Discente do curso de Biomedicina da Faculdade Única de Ipatinga (FUNIP). Email: silviamaramartins.123@gmail.com

${ }^{8}$ Orientador: Docente da Faculdade Única de Ipatinga (FUNIP). Email: adamarft@yahoo.com.br
} 
out and gaining adherents for being a non-pharmacological treatment. It consists of the stimulation of energy points, found inall parts of the human body. This stimulation allows the release of the accumulated energy flow, thus bringing relief and reduction of pain.

Keywords: Fibromyalgia. Acupuncture. Analgesia.

\section{INTRODUÇÃO}

A fibromialgia é caracterizada como uma síndrome, por estar relacionada ao número desintomas e não a um mau funcionamento específico. Tem como característica dor crônica, nãoinflamatória, sensibilizando o sistema nervoso central (SNC). O tratamento inclui o alívio dos sintomas, uma vez que sua cura é incerta. Seus principais sintomas são dores prolongadas ao longo do corpo, sensibilidade nos músculos, articulações e outros tecidos moles. Junto à dor, afibromialgia pode causar fadiga, distúrbios do sono, dores de cabeça, alterações do humor, depressão, ansiedade. Tais sintomas provocam impacto negativo na qualidade de vida (TAKIGUCHI, et al.2008).

A acupuntura tem sido reconhecida como uma modalidade terapêutica utilizada para promover ampla ação sobre as condições de dor na fibromialgia, por meio dos efeitos neurobiológicos que agem interferindo nos neurotransmissores que tenham relação com a dor.A acupuntura promove inúmeras mudanças fisiológicas no organismo humano.

No tratamentoda fibromialgia a acupuntura pode provocar analgesia, resultado da estimulação sensorial pelas agulhas, estímulo na liberação de encefalinas, serotonina e endorfinas, gerando uma resposta àdor. Ao estimular os pontos com as agulhas, há um aumento do fluxo cerebral, promovendo o controle da dor (MARCHESINI STIVAL, et al. 2014). O objetivo do presente estudo foi abordar a acupuntura como tratamento da fibromialgia.

\section{METODOLOGIA}

Este estudo trata-se de uma revisão de literatura sobre a ação da acupuntura como tratamento da fibromialgia. Para tanto, buscou-se artigos científicos publicados em revistas indexadas hospedadas em Bases de Dados da Biblioteca Virtual em Saúde (BVS - Bireme) 
com o uso mais frequente da Scielo (ScientificElectronic Library OnLine) e também o Google Acadêmico. Além dessas Bases de Dados, pesquisou-se também a Base de Dados australiana chamada PEDro (PhysiotherapyEvidenceDatabase) que indexa diversas revistas com artigos escritos para a comunidade internacional de fisioterapeutas e outros profissionais da saúde, enfatizando a fisioterapia baseada em evidência.

A escolha da Base de Dados PEDro foi devido ao fato de sabidamente a categoria profissional dos fisioterapeutas ser uma categoria mais atuante no tratamento não farmacológico das dores músculo esqueléticas. Assim, tem-se essa Base de Dados como uma importante fonte de pesquisa para os fisioterapeutas e também para os biomédicos e outros profissionais da saúde.

Os artigos científicos selecionados atenderam os critérios de inclusão estabelecidos pelos autores da pesquisa: relevância do tema, artigos de revisão, data de publicação de 2004 até 202I, no idioma português, espanhol e inglês. O intervalo entre as datas de publicação (2004 a 202I) foi devido à escassez de artigos que enfocavam a ação da acupuntura como tratamento da fibromialgia.

Os artigos foram selecionados em grupos: primeiro grupo de artigos foram aqueles quetraziam, em seu texto, apenas assuntos relacionados à fibromialgia; o segundo grupo de artigostraziam, em seu texto, apenas assuntos relacionados à acupuntura e o terceiro grupo de artigos traziam, em seu texto, a associação entre os dois assuntos: acupuntura e fibromialgia.Os artigos classificados nos grupos i e 2 foram utilizados como referencial teórico destetrabalho.

\section{DESENVOLVIMENTO}

\subsection{Acupuntura como tratamento não Famacologico da Fibromialgia}

A fibromialgia é uma síndrome idiopática que acomete sobretudo o sistema musculoesquelético. Seus sinais e sintomas estão mais relacionados a dores crônicas generalizadas em função da modificação do funcionamento dos neurônios e das vias nociceptoras. A fibromialgia é uma síndrome que pode também apresentar outros sintomas 
como fadiga, distúrbios cognitivos, alterações do humor, irritação, insônia. A fibromialgia ainda é considerada, nos dias atuais, uma síndrome incurável. Os tratamentos buscam tratar asdores e melhorar a qualidade de vida dos doentes (PEREIRA, et al. 202I).

Por meio dos critérios de diagnósticos do American College of Rheumatology (ARC), são fibromiálgicos os indivíduos que apresentam dor crônica difusa permanente há mais de trêsmeses (LORENA, et al. 2016).

Cada local ou ponto de dor é considerado um "Tender Point", termo usado para definir um ponto de intensa sensibilidade dolorosa em tecidos moles na fibromialgia que não podem ser confundidos com os "Trigger Points" ou pontos gatilhos muito comuns na síndrome dolorosa miofascial (GROSSMANN, et al. 2012; FASSICOLLO, et al. 2017).

O exame físico é um método de diagnóstico que busca a identificação dos tender points. Seu diagnóstico é eminentemente clinico, não apresentando alterações em exames laboratoriaise/ou radiológicos. Sua prevalência é de cerca de $2,5 \%$ da população mundial, acometendo principalmente indivíduos do sexo feminino (LORENA, et al. 2016).

Como uma das propostas terapêuticas para alívio da dor (analgesia) e melhora na qualidade de vida dos fibromiálgicos, tem-se a acupuntura que é um método terapêutico originado na China que consiste na estimulação de pontos cutâneos em meridianos (canais de energia) para o reestabelecimento do equilíbrio energético. Sua aplicabilidade é eficaz como tratamento coadjuvante, destacando a importância em respeitar a localização anatômica dos pontos de acupuntura (TAKIGUCHI, et al. 2008).

A acupuntura ocorre por meio de estímulos nas terminações nervosas diferenciadas da derme, tecido celular subcutâneo e músculos. Ativa o sistema de supressão de dor da medula espinhal e do cérebro, aumentando a produção de substâncias anti-inflamatórias no SNC, comoserotonina, endorfinas e encefalina. Estas substâncias possuem fortes efeitos analgésicos e suaação promove a diminuição da dor (FRANCHINI, 2013). 


\section{ACUPUNTOS}

O tratamento com medicamentos alopáticos sempre será a primeira opção para tratar a dor e o mais utilizado, porém, a possibilidade de provocar efeitos adversos e indesejáveis no organismo não pode ser menosprezado. A maioria dos pacientes desconhecem a possibilidade de outros tratamentos alternativos e coadjuvantes como a acupuntura, utilizada com sucesso para este fim, que busca a analgesia, melhora do sono, humor, fadiga (SANTOS, 2017).

No exame físico, o diagnóstico é definido com a presença de II dos I8 tender points espalhados pelo corpo. Tais pontos estão localizados na região suboccipital, músculo trapézio, região supra espinhal, altura das vértebras cervicais, articulação condrocostal, joelho, trocânter, região glútea e ao lado do cotovelo. Muitas das dores são incapacitantes devido a sua gravidade(TAKIGUCHI, et al. 2008).

A Figura I apresenta a localização dos pontos dolorosos (tender points) nos pacientes fibromiálgicos. Ressalta-se que desses 18 pontos o paciente deve apresentar dor em pelo menosir deles segundo Takiguchi et al. (2008).

Figura r: Localização dos tender points
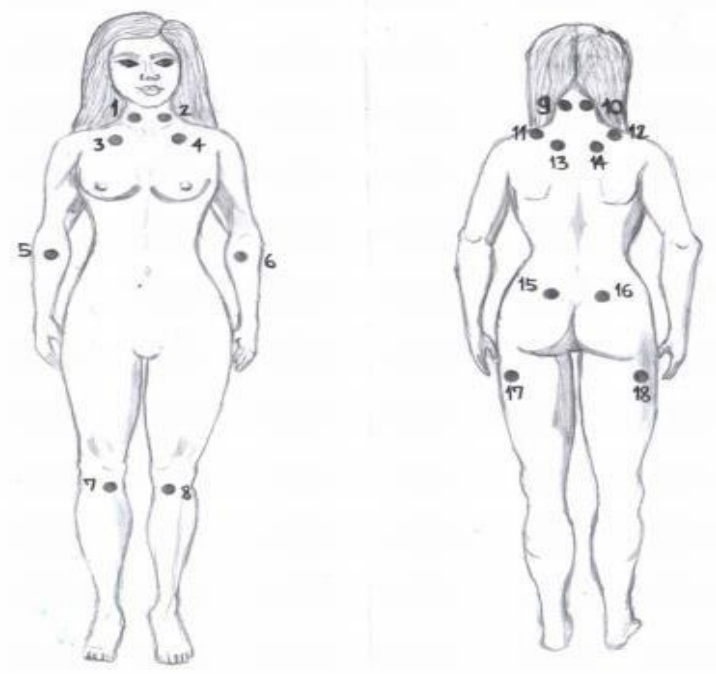

Fonte: Autores da pesquisa 
O Quadro I apresenta a localização dos acupontos em consonância com a Figura I que registra os tender points. É importante salientar que alguns acupontos podem ser coincidentes com os tender points e, também podem ser utilizados como locais de inserção da agulha, mesmo sendo um ponto doloroso (WEN, 2014).

Quadro I- Acupontos utilizados na localização dos tender points

\begin{tabular}{|c|c|}
\hline Acupontos & Localização \\
\hline I e 2 & Occipital: inserção dos músculos suboccipitais \\
\hline 3 e 4 & $\begin{array}{l}\text { Cervical baixa: anteriormente, entre os processos transversos de } \mathrm{C}_{5} \\
\mathrm{eC}_{7}\end{array}$ \\
\hline 5 e 6 & Trapézio: ponto das fibras superiores do músculo trapézio \\
\hline 7 e 8 & $\begin{array}{l}\text { Supraespinhal: inserção do músculo supraespinhal, acima da } \\
\text { espinhada escápula próximo ao ângulo medial }\end{array}$ \\
\hline 9 e io & $\begin{array}{l}\text { Segunda articulação costocondral: lateral e superiormente à } \\
\text { articulação }\end{array}$ \\
\hline II e I2 & Epicôndilo lateral: $2 \mathrm{~cm}$ distalmente ao epicôndilo \\
\hline 13 e 14 & Glúteo: quadrante superior e lateral das nádegas \\
\hline I5 e 16 & Trocânter maior: posterior à proeminência trocantérica \\
\hline 17 e 18 & Joelho: coxim gorduroso medial, próximo à linha articular \\
\hline
\end{tabular}

Fonte: Wen, 2014 
De acordo com a teoria dos cinco elementos, os pacientes diagnosticados com fibromialgia apresentam um quadro de transtorno energético no fígado, baço/pâncreas e nos rins. Este excesso de energia leva ao aparecimento dos distúrbios no organismo que se associamaos sintomas de dor crônica no sistema musculoesquelético (DA SILVA PASSOS, 2016).

A técnica da acupuntura consiste em inserir pequenas agulhas metálicas em locais específicos no corpo do paciente (acupontos), possibilitando a liberação do fluxo de energia acumulada. A sessão tem duração média de 30 a 40 minutos, compreendida desde a reavaliaçãodo paciente até a inserção e manipulação das agulhas promovendo efeitos locais e sistêmicos. Durante o tratamento, as agulhas inseridas nos acupontos podem receber estimulação manual que "transmite" esta estimulação ao local em que estão inseridas, da mesma forma, elas tambémpodem receber estímulos elétricos para aumentar a eficácia do tratamento (CLARKE, 2004).

As agulhas podem ser inseridas exatamente no local dos tender points ou em sua volta, circulando-os para que haja uma maior diminuição dos sintomas dolorosos. Quando as agulhassão posicionadas ao redor dos tender points, eletrodos do tipo "dente de jacaré” são aderidos em suas hastes para que uma corrente elétrica contínua, passe por ela e alcance o território dolorido. Vale ressaltar que o tratamento é específico para cada tipo de paciente devido a seussintomas, níveis de dor e incômodo (SANTOS, et al. 2006).

\section{CONSIDERAÇÕES FINAIS}

Dentre os tratamentos utilizados para a fibromialgia tem destaque a acupuntura. $O$ uso dessa técnica tem sido de grande auxílio e eficácia para amenizar a dor e melhorar a qualidadede vida dos pacientes. Apesar de ser uma técnica milenar e de baixo custo, uma vez que as agulhas são de preço módico, alguns pacientes ainda desconhecem sua aplicabilidade

A acupuntura faz parte das Práticas Integrativas e Complementares à Saúde (PICS) e, portanto, está previsto como tratamento no Sistema Único de Saúde (SUS) desde 2006. 
Com isto, o tratamento com a acupuntura vai aumentando seus adeptos e a técnica tende a ser cada vez mais conhecida.

Entretanto, necessário dizer que ainda falta muito para que a população em geral, tenhaconhecimento sobre o tratamento da fibromialgia pela acupuntura.

Embora a acupuntura faça parte das PICS recomenda-se uma divulgação intensa nos órgãos oficiais do governo, informando a população sobre seus benefícios e que o tratamento está acessível a todos e a todas, em especial, os fibromiálgicos.

\section{REFERÊNCIAS BIBLIOGRÁFICAS}

CLARKE T. Treatment of fibromyalgia with acupuncture and counseling. BCMJ, 2004;46:12123 Retrieved online at. Disponível em: https://bcmj.org/articles/treatment-

fibromyalgia- acupuncture-and-counseling Acesso em 23 de mar. 2021.

DA SILVA PASSOS, RAFAEL; BOAS, SARA SOUZA VILAS; PRATES, RAHAB

VIEIRA. Efeitos da acupuntura na fibromialgia: Uma revisão bibliométrica. Acta

BiomedicaBrasiliensia, v. 7, n. 2, p. 15-21, 2016.

FASSICOLLO CARLOS EDUARDO, GRACIOSA MAYLLI DAIANI, GRAEFLING

BARBARA FLISSAK, RIES LILIAN GERDI KITTEL. Temporomandibular dysfunction, myofascial, craniomandibular and cervical pain: effect on masticatory activity during rest and mandibular isometry. Rev Dor. São Paulo, 2017 jul-set;18(3):250-4

FRANCHINI, CÁSSIA DE FÁTIMA MONTEIRO. Tratamento nãofarmacológico de pacientes com fibromialgia. Brasilian Journal of Surgery and

Clinical Research, 2013.

GROSSMAN EDUARDO, TAMBARA JOSEANE STECKEL, GROSSMANN THIAGO 
KREUTZ, DE SIQUEIRA JOSÉ TADEU TESSEROLI. O uso da estimulação elétrica nervosatranscutânea na disfunção temporomandibular. Rev Dor. São Paulo, 2012 julset;13(3):271-6

LORENA, SUÉLEM BARROS DE LORENA, EDUARDO AUGUSTO DOS SANTOS PIMENTEL, VANESSA MEDEIROS FERNANDES, MÁRCIO BOTELHO PEDROSA,ALINE RANZOLIN, ANGELA LUZIA BRANCO PINTO DUARTE. Avaliação de dor e

qualidade de vida de pacientes com fibromialgia. Revista dor, v. I7, n. I, p. 8-ıI, 2016.

MARCHESINI STIVAL, REBECCA SARAY MARCHESINI STIVAL, PATRÍCIA RECHETELlO CAVAlHEIRO, CAMILA STASIAK, DAYANA TAlitA GALDINO, BIANCA ELIZA HOEKSTRA, MARCELO DERBLI SCHAFRANSKI. Acupuntura na

fibromialgia: um estudo randomizado-controlado abordando a resposta imediata da dor. Revista Brasileira de Reumatologia, v. 54, n. 6, p. 431-436, 2014.

PEREIRA, HELOÍSA SALVADOR DOS SANTOS PEREIRA, MARIANGELA DASILVA NUNES, CAÍQUE JORDAN NUNES RIBEIRO, MARIA DO CARMO DE

OLIVEIRA RIBEIRO. Efeitos da acupuntura na fibromialgia: revisão integrativa. BrJP, n.AHEAD, 202I.

SANTOS, A. M. B. ASSUMPÇÃO A, MATSUTANI LA, PEREIRA CAB, LAGE LV, MARQUES AP. Depressão e qualidade de vida em pacientes com fibromialgia. BrazilianJournal of Physical Therapy, v. Io, n. 3, p. 317-324, 2006. 
crônica esua aplicabilidade no Sistema Único de Saúde: Revisão da literatura. 2017.

TAKIGUCHI, RAYMOND S. TAKIGUCHI, VANESSA SATIE FUKUHARA, JULIANAFERREIRA SAUER, ANA ASSUMPÇÃO, AMÉLIA PASQUAL MARQUES. Efeito da

acupuntura na melhora da dor, sono e qualidade de vida em pacientes fibromiálgicos: estudopreliminar. Fisioterapia e Pesquisa, v. 15, n. 3, p. 280-284, 2008.

WEN, TOM SINTAN. Acupuntura clássica chinesa. 2 ed., 3 reimpr. São Paulo; Cultrix, 2014.248p. 